\title{
Study on low-power Sensor and Heater for PCR microchip
}

\author{
Wenbo He ${ }^{*}$ Jinyu Liu ${ }^{[0000-0003-0233-3493]}$, Lei Fan ${ }^{[0000-0003-4654-6511] ~}$ \\ School of Information Science and Engineering, Dalian Polytechnic University, \\ Dalian 116034, China \\ hewb@dlpu . edu. cn
}

\begin{abstract}
Low-power temperature sensor and heater were designed and fabricated by using chromium, nickel chromium alloy $(\mathrm{Ni}: \mathrm{Cr}=80 \%: 20 \%)$ and nickel materials respectively. Through the analysis of the working characteristics of the Low-power temperature sensor and heater, it is shown that the $\mathrm{Ni} / \mathrm{Cr}$ film resistance heater and the nickel film resistance temperature sensor can meet the requirements of the heating rate and temperature consistency of the reaction chamber for PCR amplification.
\end{abstract}

Keywords: PCR microchip, Low-power temperature Sensor, Low-power heater, micro-fabrication, film-resistance.

\section{Introduction}

In 1985, K. B. Mullis and R. K. Saiki of Cetus Company, USA, invented a specific DNA amplification technique in vitro. The essence of this technique is the enzymatic synthesis of specific DNA fragments in vitro. This technique is called Polymerase Chain Reaction, or PCR for short. PCR is an enzymatic synthesis reaction dependent on DNA polymerase in the presence of polymerase, primers and four deoxyribonucleotides. It works through three steps: high temperature denaturation of doublestranded DNA $\left(92-94^{\circ} \mathrm{C}\right)$, low temperature annealing of primers and templates $(37-$ $55^{\circ} \mathrm{C}$ ), and primer extension under suitable temperature conditions $\left(68-72^{\circ} \mathrm{C}\right)$. Through repeated cycles of these three steps, PCR can achieve exponential amplification of specific DNA sequence yield with the number of cycles, thus achieving the goal of rapid and large-scale amplification of template DNA [1-2]. Not long after the advent of PCR amplification technology, it quickly became the basic technology and powerful tool of molecular biology research because of its simple, fast and efficient characteristics, and has been widely used in medicine and biology.

Subsequent The traditional PCR chip amplification reaction vessel and heater are separated [3], the volume is huge, the operation is inconvenient, and the sample consumption is large. Therefore, the traditional PCR chip can not meet the needs of modern biochemical research. In addition, mature thin-film technology and microfabrication technology make it possible to integrate reaction vessel, heater, temperature sensor and other components into a chip. PCR biochip is an integrated PCR microreactor, 
which is made on silicon, glass and organic polymers by using the technology of micro-electro-mechanical system (MEMS) [4]. It can control the temperature of reaction zone accurately by using heaters and temperature sensors integrated on the chip. It fully embodies the advantages of microchip, such as high efficiency, rapidity and saving reagents.

In this paper, the characteristics of a self-developed low-power temperature sensor and low-power heater for integrated PCR chip are analyzed.

\section{Development of Low-power temperature Sensor and heater}

There are two main types of PCR microchip fabricated by MEMS technology: the first is the single reaction zone structure, in which the whole process of amplification is carried out in one reaction chamber, and the continuous cycle of PCR amplification is realized by periodically changing the temperature in the reaction cell. The second is the three reaction zone structure, i.e. the amplification reaction is carried out in three different reaction zones, each of which maintains a constant temperature. Under the action of external forces, the sample flows through the channel on the chip in three reaction zones to realize the transformation of the sample in three temperature zones, thus realizing the cycle of PCR. In single reaction region PCR chips, the rate of temperature change may affect the amplification effect, but there is no such problem on three reaction region PCR chips. However, the fabrication process of three reaction zone PCR chip is much more complicated than that of single reaction zone PCR chip. How to achieve thermal isolation between different temperature zones and how to accurately control the temperature of the reaction zone are the problems that must be solved by the three reaction zone PCR chip.

On the basis of consulting the literature [5-6], according to the working requirement of the integrated PCR chip and the problems faced in the current research, we have developed a single reaction region integrated PCR chip. Fig.1 shows a schematic diagram of an integrated PCR chip.

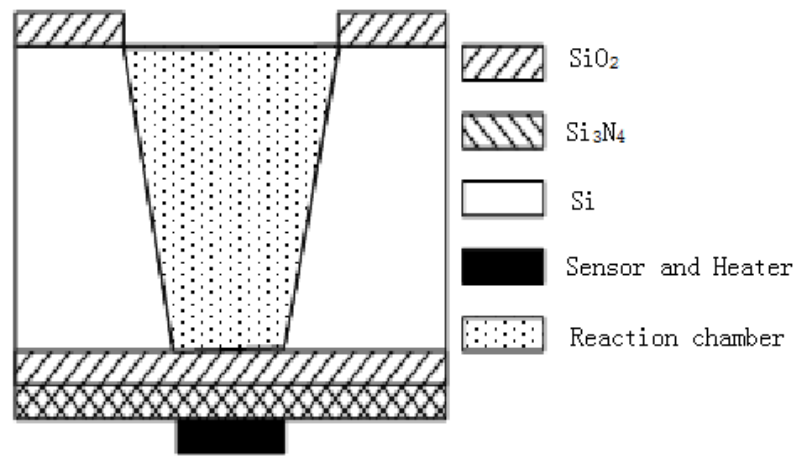

Fig. 1. Schematic structure of integrated PCR chip. 
As the main device for DNA sample amplification, the PCR chip must be able to meet the thermal uniformity, the accuracy of temperature control and the accuracy of physical size. Therefore, the material selection and structure design of low-power temperature sensor and heater are important factors to determine the performance of the PCR chip.

\subsection{Material selection of Low power temperature Sensor and heater}

At present, the most suitable material for making thin film resistance temperature sensor and heater is platinum, which has good heating and temperature characteristics [7]. But because platinum is a precious metal, the cost is too high, so this paper hopes to use some cheaper metals to make temperature sensor and heater.

Based on the literature [8-10], it is found that chromium and nickel-chromium alloys are good heating materials, and nickel is a good temperature sensor material. In this paper, we hope to use the same material to make temperature sensor and heater, because the reduction of process means the increase of chip yield. Therefore, chromium $(\mathrm{Cr})$, nickel-chromium alloy ( $\mathrm{Ni}: \mathrm{Cr}=80 \%: 20 \%)$ and nickel $(\mathrm{Ni})$ are used to make heaters and temperature sensors respectively.

\subsection{Design of Low-power temperature Sensor and heater}

In this paper, four independent PCR reaction chambers are etched on a single crystal silicon of type $\mathrm{N}$ with a crystal orientation of [100] and a size of $20 \times 15 \mathrm{~mm}^{2}$ at one time.

In order to improve heating efficiency and ensure accurate temperature control, the area of Low-power heater at the bottom of the reaction chamber should be enlarged as much as possible so that the reaction chamber can be heated uniformly. Meanwhile, the Low-power temperature sensor should be placed in the middle of the reaction chamber bottom.

Fig. 2 shows the structure of the low-power temperature Sensor and heater designed in this paper. The longer resistance is the heater and the shorter resistance is the temperature sensor. The line width of the thin film resistance is $50 \mu \mathrm{m}$, the distance between the lines is $100 \mu \mathrm{m}$, and the area of the welding pad at the top of the resistance bar is $200 \times 200 \mu \mathrm{m}^{2}$.

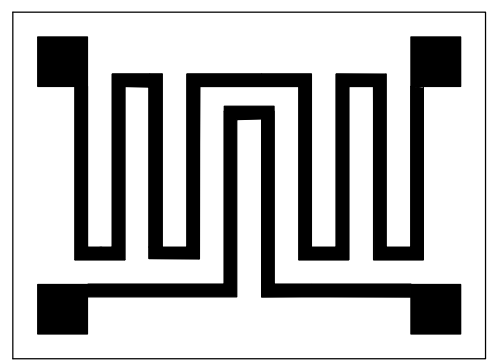

Fig. 2. Structure of Low-power temperature Sensor and heater. 


\subsection{Fabrication of Low-power temperature Sensor and heater}

The fabrication of low-power temperature Sensor and heater is combined with the fabrication of PCR chip. Based on a large number of references and previous work experience in the laboratory, wet etching and sputtering process is adopted. The process flow is as follows:

According to the design scheme, the required photolithographic mask are obtained by drawing graphics on coordinate paper, carving red film, exposure, development and stereotyping.

$\mathrm{SiO}_{2}$ thin films with thickness of about $1 \mu \mathrm{m}$ are grown on both sides of single crystal silicon by thermal oxidation method, and then $\mathrm{a} \mathrm{Si}_{3} \mathrm{~N}_{4}$ thin film with thickness of about $1840 \mathrm{~nm}$ is deposited on one side of $\mathrm{SiO}_{2}$ by LPCVD method as an insulating layer.

On the side without $\mathrm{Si}_{3} \mathrm{~N}_{4}$ film, the window of PCR reaction chamber is engraved on $\mathrm{SiO}_{2}$ using photolithographic mask.

Using $\mathrm{SiO}_{2}$ as mask and $\mathrm{KOH}$ solution as anisotropic etchant, the reaction chamber of PCR is etched on silicon wafer.

Because of the incompatibility between silicon and PCR, a thin $\mathrm{SiO}_{2}$ film is grown on the inner wall of the reaction chamber by thermal oxidation.

At the bottom of the reaction chamber, a thin film heater and a thin film temperature sensor are fabricated as follows: first, a thin metal film is sputtered on $\mathrm{Si}_{3} \mathrm{~N}_{4}$ by vacuum sputtering method, then the metal film is lithographed by photolithographic mask, then corroded by corrosive solution, and finally annealed in a nitrogen-filled environment at $400^{\circ} \mathrm{C}$ for 30 minutes.

Pressure solder joint: a layer of metal aluminum is grown on the metal layer by vacuum evaporation coating machine, photolithographic mask is used to photolithograph it, then corrode it with corrosive solution. Finally, it is placed in a nitrogenfilled environment at $500^{\circ} \mathrm{C}$ and alloyed for $10-15$ minutes to ensure good ohmic contact between silicon and metal.

The alloy film is bonded by hot pressing with a gold wire ball welding machine to connect the external lead.

\section{Characteristic analysis of Low-power temperature Sensor and heater}

According to the application requirements, it is hoped that the thin film resistance heater has high heating efficiency, and the resistance of the thin film temperature sensor varies sensitively with the change of the external environment. The working characteristics of the low-power temperature sensor and the low-power heater made of three materials will be analyzed separately. 


\subsection{Analysis of temperature characteristics}

According to the temperature requirement of PCR amplification reaction, the analysis range of heater and temperature sensor is chosen in the range of $30 \sim 110^{\circ} \mathrm{C}$.

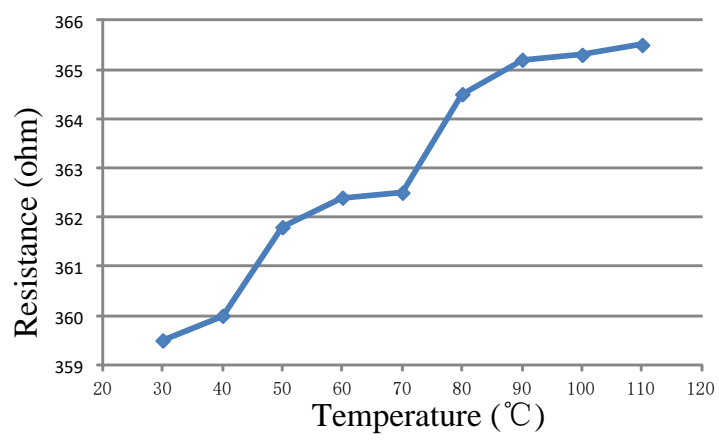

Fig. 3: Temperature Characteristic of integrated $\mathrm{Cr}$ resistance.

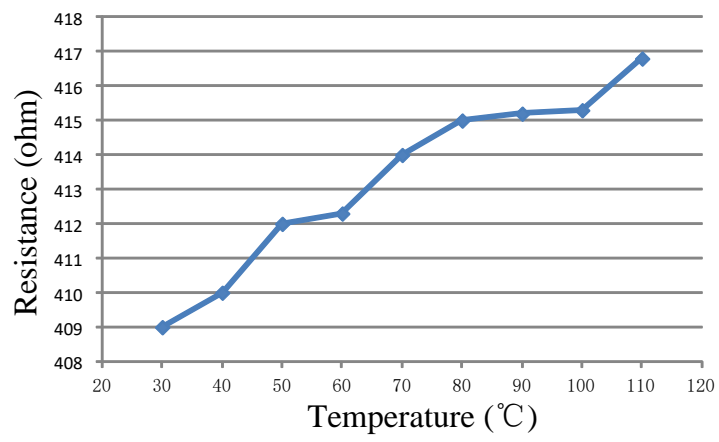

Fig. 4: Temperature Characteristic of integrated $\mathrm{Cr}$ resistance.

Fig. 3 and Fig. 4 show the temperature characteristic curves of two $\mathrm{Cr}$ film resistances. The abscissa is the test temperature and the ordinate is the resistance value. It can be seen from the figure that the $\mathrm{Cr}$ film resistance has no linear temperature characteristic in the temperature range of $30-110^{\circ} \mathrm{C}$, and is insensitive to temperature. The resistance value changes within several ohms with the change of $10^{\circ} \mathrm{C}$, so the chromium film resistance can not be used as temperature sensor and heater.

Fig. 5 and Fig. 6 show the temperature characteristic curves of the two nickel thin film resistances. The abscissa is the test temperature and the ordinate is the resistance value. It can be seen that the resistances of nickel film has good linear temperature characteristics in the temperature range of $30-110^{\circ} \mathrm{C}$, and is very sensitive to temperature. The variation of resistor is tens of ohms for every $10^{\circ} \mathrm{C}$ change of temperature. This shows that the resistance of nickel film is a good temperature sensor, but it is too sensitive to temperature, therefore, it can not be used as a heater. 


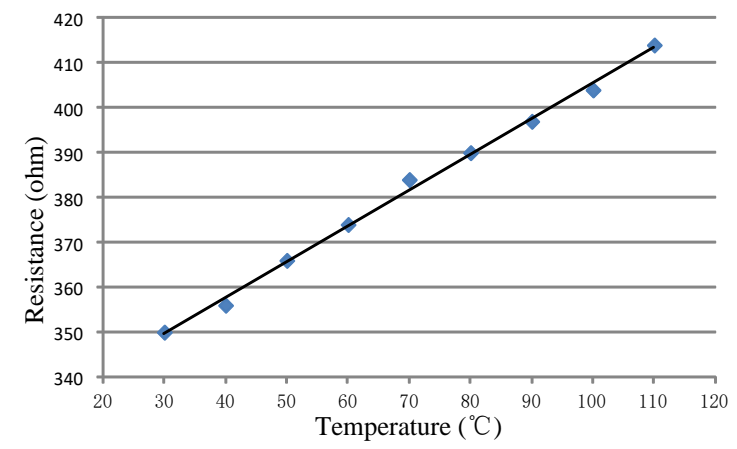

Fig. 5. Temperature Characteristic of integrated Ni resistance.

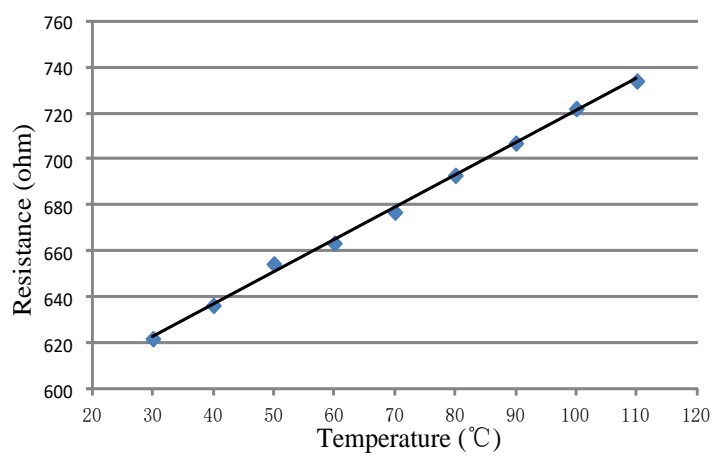

Fig. 6. Temperature Characteristic of integrated Ni resistance.

The temperature characteristic curve of Ni-Cr alloy film resistance is no longer given here as its resistance value hardly varies with temperature. The values of two thin film resistances are $1100 \mathrm{ohms}$ and $428 \mathrm{ohms}$ at $20^{\circ} \mathrm{C}$ respectively. The values of two thin film resistances are $1102 \mathrm{ohms}$ and $430 \mathrm{ohms}$ at $100^{\circ} \mathrm{C}$ respectively. This shows Ni$\mathrm{Cr}$ alloy is a good heating material, but it can not be used to make temperature sensor.

\subsection{Analysis of Heating Characteristics}

In order to verify whether the nickel chromium alloy thin film resistance heater can meet the needs of PCR amplification reaction, the heating effect of its PCR chip is tested by micro thermocouple.

Fig. 7 shows the Heating characteristic of the integrated $\mathrm{Ni} / \mathrm{Cr}$ resistance. The resistance value tested is $1281 \mathrm{ohms}$. The abscissa is the applied voltage, and the ordinate is the highest temperature that can be reached in the reaction chamber of the PCR. It can be seen from the figure that the heating effect is very poor when the applied voltage is low. However, when the applied voltage exceeds a certain critical 
value, the heating effect varies greatly, and the maximum temperature in the reaction chamber increases approximately linearly with the increase of the applied voltage. When the applied voltage is $48 \mathrm{~V}$, the highest temperature in the reaction chamber reaches $116^{\circ} \mathrm{C}$, while the temperature required for PCR amplification is below $100^{\circ} \mathrm{C}$, which indicates that the heater can meet the requirements of the temperature of PCR amplification.

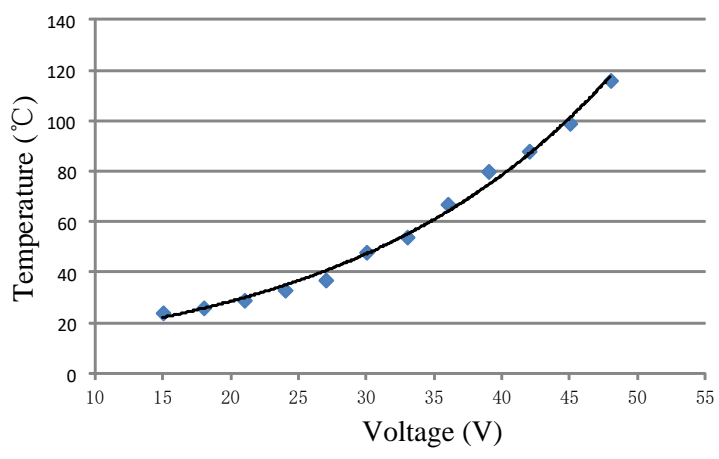

Fig. 7. Heating Characteristic of $\mathrm{Ni} / \mathrm{Cr}$ resistance.

The amplification reaction of PCR is repeated in three temperature zones, so the heating speed of single reaction zone PCR chip is very high, and generally requires heating speed not less than $0.5^{\circ} \mathrm{C} / \mathrm{s}$. In order to verify whether the resistance of nickelchromium alloy thin film can meet the requirement of heating rate for PCR amplification reaction, the heating states of two thin film resistors are tested. Fig. 8 and Fig. 9 show temperature-time characteristic curves of thin film resistors with resistance values of $1281 \mathrm{ohms}$ and $504 \mathrm{ohms}$ respectively. The abscissa is the heating time and the ordinate is the temperature reached.

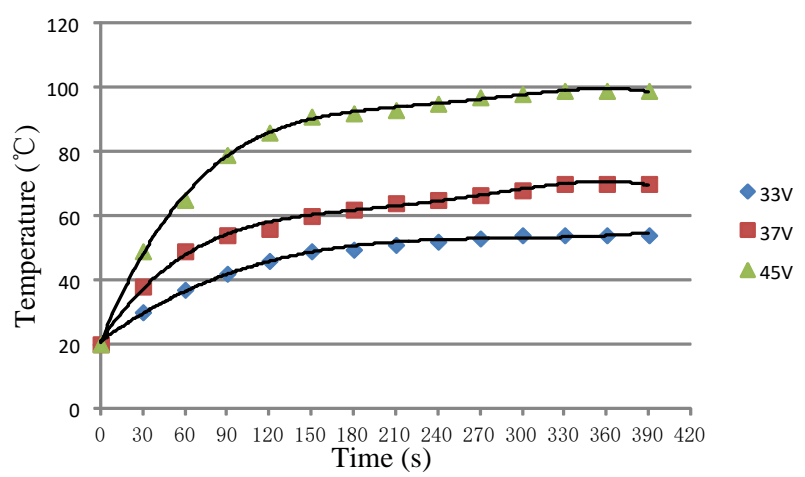

Fig. 8. Heat characteristic of $\mathrm{Ni} / \mathrm{Cr}$ resistance. 


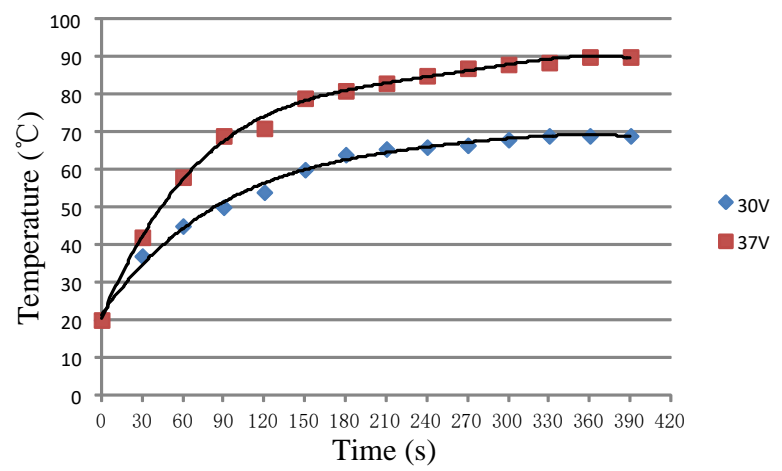

Fig. 9. Heat characteristic of $\mathrm{Ni} / \mathrm{Cr}$ resistance.

As can be seen from Fig. 8 and Fig. 9, the heating characteristics of the two heaters are comparatively consistent. The temperature rises rapidly in the first 30 seconds. After 30 seconds, the temperature rises slowly. After 90 seconds, the temperature changes more slowly, and gradually tends to a constant temperature. At the same time, with the increase of the applied voltage, the maximum temperature of the reaction chamber can also change significantly. In addition, when the applied voltage is the same, the heating effect of the heater with small resistance is obviously better than that of the heater with large resistance, and the stable temperature can be achieved is also high. For example, when the applied voltage is $37 \mathrm{~V}$, the final constant temperature of small resistance is $94^{\circ} \mathrm{C}$, while the final constant temperature of large resistance is $74^{\circ} \mathrm{C}$. Within 30 seconds, the average temperature change speed of small resistance is $0.75^{\circ} \mathrm{C} / \mathrm{s}$, while that of large resistance is $0.5^{\circ} \mathrm{C} / \mathrm{s}$. This also provides a train of thought for future research, which means we can use multiple small resistances to replace the large snake resistance.

Based on the above analysis, the Ni-Cr alloy film resistance heater can meet the needs of the temperature change of the PCR amplification reaction, and can be used as the heater of the PCR microchip.

\subsection{Analysis of Temperature Uniformity}

In order to test the temperature uniformity in the reaction chamber, the temperature is measured by sticking four micro thermocouples to the bottom of the reaction chamber. The temperature-time characteristics of the four test points are shown in Fig. 10. The film resistance value used is $1281 \mathrm{ohms}$, and the applied voltage is $45 \mathrm{~V}$. The abscissa is the heating time and the ordinate is the temperature reached.

From Fig.10, it can be seen that the temperature uniformity in the reaction chamber is very good, and the temperature of each point is comparatively consistent. This shows that the heater designed in this paper is reasonable and can meet the requirements of temperature uniformity for PCR amplification reaction. 


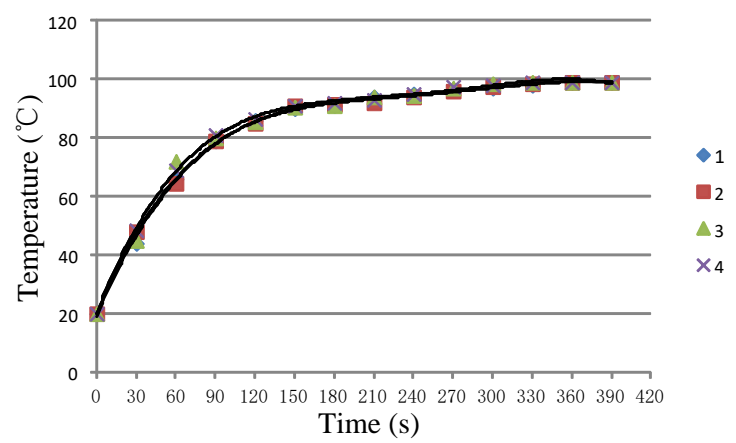

Fig. 10. Temperature characteristics of the four test points.

\section{Conclusions}

In this paper, a single reaction zone integrated PCR chip is designed and fabricated. The low-power temperature Sensor and heater are made of chromium, $\mathrm{Ni}-\mathrm{Cr}$ alloy (Ni: $\mathrm{Cr}=80 \%: 20 \%)$ and nickel respectively. The low-power temperature Sensor and heater are integrated at the bottom of the PCR reaction chamber. The low-power temperature Sensor and heater depend on the process to some extent. The resistance deviation and consistency can be further controlled by optimizing the design parameters and process parameters. The results show that the nickel-chromium alloy film resistance produced in this paper can meet the requirements of heating speed and temperature consistency of PCR reaction chamber, and can be used as heater of PCR microchip. Nickel film resistance can be used as temperature sensor of PCR microchip.

In the process of chip fabrication, using different materials to make heaters and temperature sensors means the process is more troublesome and material consumption is greater. Therefore, we still hope to find the same material to fabricate heaters and temperature sensors.

\section{References}

1. Giordano, B. C. J., Swedberg, S., Huhmer, A. F. R., Landers, J. P.: Polymerase Chain Reaction in Polymeric Microchips: DNA Amplification in Less Than 240 Seconds, Analytical Biochemistry, vol. 291, pp. 124-132(2001)

2. Cheng, J., Shoffner, M. A., Hvichia, G. E.: Investigation of different PCR amplification system in microfabricated silicon-glass chips, Nucleic Acids Research, 24(2): 380385(1996)

3. Nagai H., Murakami, Yuji, Yokoyama K., Tamiya E, : High-throughput PCR in silicon based microchamber array, Biosensors and Bioelectronics, 16 (9-12): 1015-1019(2001)

4. Sun, K., Yamaguchi, A., Ishida, Y.: A heater-integrated transparent microchannel chip for continuous-flow PCR [J], Sensors and Actuators: B, 87(2/3): 612-619 (2002) 
5. Yu. C. Y., Liang. W. S.: Fabrication and characterization of a flow-through PCR device with integrated chromium resistive heaters[J].Journal of the Chinese Institute of Chemical Engineers(3/4), 333-339(2007)

6. Lai, L. F., Sun, R.: Processing technology of embedded thin-film resistor materials.In:2011 International Symposium on APM, pp.60-65.XiaMen(2011)

7. Lai, L. F., Su, X.S., Fu, X. Z., Sun, R.: The microstructure and properties of C and W codoped $\mathrm{Ni} / \mathrm{Cr}$ embedded thin film resistors, Surface Coat. Technol, vol. 259, pp. 759766( 2014)

8. Yan, J. W., Zhou, J. ch.: The oxidation and the electrical properties of Ni-Cr thin film after rapid thermal annealing, Int. J. Mod Phys B, Vol. 21, No. 26, pp. 4561-4574(2007)

9. Danisman, M., Cansever, N.: Effect of $\mathrm{Cr}$ content on mechanical and electrical properties of Ni-Cr thin film, J. Alloys Compd, vol. 493, pp. 649-653( 2010) 